\title{
Modulation of Visceral Sensitivity by Faecal Microbiota Transplantation (FMT): The Active Role of Gut Microbiota in the Persistence of Abdominal Pain
}

\section{Elena Lucarini}

Universita degli Studi di Firenze

Vincenzo Di Pilato

Universita degli Studi di Genova

Carmen Parisio

Universita degli Studi di Firenze

Laura Micheli

Universita degli Studi di Firenze

Alessandra Toti

Universita degli Studi di Firenze

Alessandra Pacini

Universita degli Studi di Firenze

Gianluca Bartolucci

Universita degli Studi di Firenze

Simone Baldi

Universita degli Studi di Firenze

\section{Elena Niccolai}

Universita degli Studi di Firenze

\section{Amedeo Amedei}

Universita degli Studi di Firenze

Gian M. Rossolini

Azienda Ospedaliero Universitaria Careggi

Claudio Nicoletti

Universita degli Studi di Firenze

John F. Cryan

University College Cork National University of Ireland

Siobhain M. O'Mahony

University College Cork National University of Ireland

Carla Ghelardini

Universita degli Studi di Firenze 


\section{Research}

Keywords: microbiota-gut-brain axis, visceral pain, IBS, IBDs, faecal microbiota transplantation

Posted Date: August 31st, 2020

DOI: https://doi.org/10.21203/rs.3.rs-66878/v1

License: (c) (1) This work is licensed under a Creative Commons Attribution 4.0 International License. Read Full License 


\section{Abstract}

Background: Recent findings linked gastrointestinal disorders characterized by abdominal pain to gut microbiota composition. The present work aimed to evaluate the power of gut microbiota as a visceral pain modulator and, consequently, the relevance of its manipulation as a therapeutic option in reversing the persistence of visceral hypersensitivity consequent to colitis induced by the intra-rectal injection of 2,4-dinitrobezenesulfonic acid (DNBS) in rats.

Results: The effect of faecal microbiota transfer (FMT) from viscerally hypersensitive DNBS and naïve donors was evaluated in control rats after an antibiotic-mediated microbiota depletion. FMT from DNBS donors induced a long-lasting visceral hypersensitivity in control rats. Pain threshold trend correlated with major modifications in the composition and structure of the gut microbiota at phylum (Proteobacteria and Firmicutes to Bacteroides ratio) and family levels (Enterobacteriaceae, Akkermansiaceae and Lachnospiraceae). Acetic acid was significantly increased in the recipients FMT from DNBS donors. Gut cytokine profile, as well as tryptophan metabolism were similarly altered after FMT from both DNBS and naïve donors. By contrast, no significant alterations of colon histology, permeability and monoamines levels were detected. Finally, following FMT from healthy donors to DNBS-treated animals, a counteraction of persistent visceral pain was achieved.

Conclusions: The present results provide novel insights into the relationship between intestinal microbiota and visceral hypersensitivity, highlighting the therapeutic potential of microbiota modulation on persistent abdominal pain.

\section{Background}

Visceral pain management is a major clinical problem, given the lack of effective and safe drugs [1]. The development of a chronic visceral hypersensitivity frequently occurs in patients with history of intestinal damage. Indeed $20-50 \%$ of patients affected by Inflammatory Bowel Diseases (IBDs) manifest a chronic abdominal pain also in the remission phase of colitis. On the other hand, an acute gut illness increases the risk of developing the Irritable Bowel Syndrome (IBS) by 7-fold [2]. Several mechanisms have been proposed to contribute to the initiation, exacerbation and persistence of visceral pain. Among them there is dysbiosis of gut microbiota [3-5]. The most well-documented line of evidence linking loss of gastrointestinal microbial homeostasis to the development of chronic visceral hypersensitivity comes from the literature on post-infectious IBS [6]. Consistently, the disturbance of the gut microbiota in earlylife [7] as in adulthood affects the local immune response and enhances pain signalling in rodents [810]. The brain, the gut microbiota and the immune system show reciprocal associations in health and disease [11]. The brain, via the autonomic nervous system and the hypothalamic-pituitary-adrenal axis, influences intestinal functions and gut microbial composition [12]. On the other hand, microbiota alterations can influence brain structure and function either developmentally or in response to acute perturbations, setting a regulatory loop between the gut and the brain $[13,14]$. Although alterations of the gut microbiota composition and/or metabolism have been associated with intestinal inflammation [15], a 
definitive cause-effect relationship between dysbiosis and post-inflammatory visceral pain persistence has not yet been determined. The aims of the present work were i) to evaluate the effect of Faecal Microbiota Transfer FMT from viscerally hypersensitive donors in remission from colitis induced by 2,4dinitrobezenesulfonic acid (DNBS) on controls visceral sensitivity, ii) to study the mechanisms by which the gut microbiota can directly affect pain perception, and iii) to evaluate the therapeutic efficacy of healthy FMT on persistent post-inflammatory visceral pain.

\section{Results}

\section{FMT from DNBS donors transfers visceral hypersensitivity}

To limit the effects of the antibiotics on visceral sensitivity, we investigated the minimum treatment duration needed to obtain a sufficient microbiota depletion prior to proceed with FMT. The culture-based analyses performed on freshly collected faecal pellets showed no viable bacterial cells after 7 days treatment. On day 7, antibiotics induced visceral hypersensitivity as assessed by abdominal withdrawal response (AWR) to colorectal distension (CRD) (Fig. 1A), whereas the viscero-motor response (VMR) was unaltered (Fig. 1B). Antibiotic-induced hypersensitivity was mild on day 12 and completely disappeared on day 18. Starting on day 7 and following the scheme reported in Fig. 1 FMT was performed using faeces from control (FMT ${ }^{\text {CTR }}$ ) or from animals in which a severe visceral pain was induced by the intrarectal injection of DNBS (FMT $\left.{ }^{\mathrm{DNBS}}\right)$.

FMT $^{\text {DNBS }}$ induced, in comparison to FMT ${ }^{\mathrm{CTR}}$, a significant increase of both AWR and VMR to CRD after the set I of FMT (days 12-18, Fig. 1A and B, respectively). Hypersensitivity was consolidated after the set II of FMT (days 25-32, Fig. 1), in particular when evaluated as AWR in awake animals (until day 39; Fig. 1A). Three weeks after the interruption of treatments (day 46), the effect of FMT ${ }^{\mathrm{DNBS}}$ ended and the animals' visceral sensitivity came back to the value registered in controls (Fig. 1A). FMT ${ }^{\text {CTR }}$ did not induced significant effects on pain threshold, showing no differences in comparison to the group abx + vehicle.

We observed similar results in preliminary experiments employing a longer antibiotic treatment (24 days), in this case though the effect of microbiota transplantation on visceral sensitivity was less clear due to the persistence of antibiotics-induced gut sensitivity alterations (Figure S1).

\section{Visceral hypersensitivity is accompanied by alteration of the gut microbiota composition}

The taxonomic analysis of faecal suspensions, subsequently used for FMT, revealed several differences between control and DNBS-treated animals (Figure S3A). For instance, the phylum Verrucomicrobia showed diminishing trend in suspensions from DNBS-treated animals used for the FMT. At lower taxonomic level, the Prevotellaceae family was almost absent in suspensions from DNBS-treated animals, while other families were found to be underrepresented, including Akkermansiaceae and 
Lactobacillaceae, on the other hand, an expansion of the Bacteroidaceae and Tannerellaceae families was identified (Figure S3A).

Analysis of alpha diversity in samples collected after the I and II set of FMT (day 18 and 46), showed a significantly greater microbial diversity in samples from the groups of animals receiving abx + FMTCTR and $a b x+F M T^{D N B S}$ compared to others (Figure S3B). This observation suggested a stable engraftment of gut microbiota following transplantation, whereas an antibiotic-driven dysbiosis was still persisting in animals of the $a b x+$ vehicle group. Such differences were even more marked on day 46 , where the II set of FMT was clearly associated with a higher microbial diversity in samples from abx + FMT ${ }^{\text {CTR }}$ and abx + FMT ${ }^{\text {DNBS }}$ compare to controls. The long-lasting effect of the antibiotic and antifungal treatment was still evident in samples from animals belonging to the abx + vehicle group that displayed an overall lower microbial richness compared to other groups (Figure S3B). On day 32, samples from the abx +FMTDNBS group were characterized by a microbial richness slightly higher, although not significant, than samples from abx $+\mathrm{FMT}^{\mathrm{CTR}}$. Evaluation of beta-diversity through PCoA using the Bray-Curtis metric (Fig. 2A) showed that samples from the abx + vehicle group clustered away from samples of corresponding controls (i.e. controls at day 18 and 46), showing a different composition of the gut microbiota. Defined clusters of samples belonging to the $a b x+F M T^{C T R}$ and $a b x+F M T^{D N B S}$ groups were identifiable on PCoA plots on day 18 (PERMANOVA, $p=0.03$; test statistic, 4.49), day 32 (PERMANOVA, $p=0.04$; test statistic, 5.70 ) and day 46 (PERMANOVA, $p=0.02$; test statistic, 3.54), with a more pronounced group separation occurring on day 32 according to the Bray-Curtis metric (Fig. 2B). A similar behaviour was observed in PCoA plots using the weighted UniFrac metric (Figure S4B). Consistently with the sample clustering observed on beta-diversity PCoA plots, analysis of the taxonomic composition of samples belonging to the $a b x+F M T^{C T R}$ and $a b x+F M T^{D N B S}$ groups pointed out to several significant differences at phylum and family level.

In detail, while no major changes in the relative abundance of most represented phyla were detected at day 18, an expansion of the Lactobacillaceae and Staphylococcaceae families was observed in samples from the $a b x+F M T^{D N B S}$ and from the $a b x+F M T^{C T R}$ groups (Kruskal-Wallis $p<0.043$ ), respectively (Fig. 2C). At day 32, marked changes were identified in samples from the $a b x+F M T^{D N B S}$ at a phylum level, showing a higher Firmicutes to Bacteroidetes ratio and a contraction of Proteobacteria and Actinobacteria (Kruskal-Wallis $\mathrm{p}=0.02$ ); at family level, the most noticeable change consisted in a large expansion of Lachnospiraceae and a concomitant contraction of Enterobacteriaceae and Burkholderiaceae in samples from the $a b x+F M T^{\text {DNBS }}$ (Kruskal-Wallis $p=0.02$ ). Additional statistically significant variations (Kruskal-Wallis $p<0.047$ ) have been observed in samples from the abx $+F_{M T}{ }^{D N B S}$ and from the $a b x+F M T^{C T R}$ groups at day 32, although occurring at a lower extent (Fig. 2B and C). At day 46 , some of the previously observed signatures were still present in samples from the $a b x+F M T^{D N B S}$ group, as the higher Firmicutes to Bacteroidetes ratio, an even more pronounced expansion of the Lachnospiraceae family and a contraction of Enterobacteriaceae and Burkholderiaceae relative abundances (Kruskal-Wallis $\mathrm{p}<0.043$ ) (Fig. 2B and C). Interestingly, a peculiar variation trend was 
observed in the relative abundance of Akkermansiaceae, being overall equally represented in samples from the $a b x+F M T^{C T R}$ and $a b x+F M T^{D N B S}$ groups at Day 18 and 46 and almost disappeared in samples from the $a b x+F M T^{\text {DNBS }}$ group at Day 32 (Fig. 2C).

\section{Changes in the levels of faecal SCFAs are associated with visceral hypersensitivity}

Faecal pellets were collected from animals undergoing to FMTs 7 days after the I and the II set of treatment (days 18 and 32) and 3 weeks after the last treatment (day 46). An increase of the total amount of SCFAs was found in the $a b x+F M T^{\text {DNBS }}$ group in respect to the $a b x+F M T^{C T R}$. This difference was detectable already on day 18 , becoming significant on day 32 and extinguished on day 46 (Fig. 3A). Further in-depth analysis revealed that alteration on day 18 were due to an overall increase of fatty acids, while on day 32 acetic acid alone was found to be raised, while butyric acid significantly decreased (Fig. 3B, C, D).

Correlation analyses between concentration of SCFAs and relative abundances of microbial taxa that were previously found to statistically differ in $a b x+F M T^{C T R}$ and $a b x+F M T^{D N B S}$ groups revealed that some time-dependant associations were identifiable. In detail, after the I set of FMT at day 18, Peptococcaceae and Clostridiales vadin BB60 group were found to be negatively correlated with butyric acid (Spearman $r$ range: $-0.857,-0.821 ; P<0.035$ ), while the Lactobacillaceae family was positively correlated with acetic acid (Spearman r: $+0.850, \mathrm{P}: 0.02$ ). After the II set of FMT at day 32 , several bacterial families were found to be negatively correlated with acetic acid (Spearman $r$ range: -0.778 , -0.881; $\mathrm{P}<0.030$ ), including Burkholderiaceae, Enterobacteriaceae, Bifidobacteriaceae, Enterococcaceae, Mollicutes RF39, as well as two phyla including Actinobacteria and Proteobacteria (Spearman r range: $-0.928,-0.833 ; \mathrm{P}<0.015)$. On day 46, Rikkenellaceae and Anaeroplasmataceae were positively correlated with iso-Butyric acid (Spearman $r$ range: $0.898,0.803 ; P<0.025)$

\section{FMT including the source does not affect colon histology}

Colon samples were collected on days 7 ( $24 \mathrm{~h}$ after the end of antibiotic treatment), 32 (when the effect of FMT on pain was well established) and 46 (when the effect of FMT extinguished). The Macroscopic Damage Score (MDS; Fig. 4A) highlights the presence of hyperaemia and a slight thickening of wall after the antibiotic treatment (day 7). These results were confirmed by microscopic analysis: an infiltration of neutrophils in mucosa and sub-mucosa were revealed in the animals receiving the antibiotic treatment (day 7; black arrow; Fig. 4B). The tissue alteration disappeared following to the interruption of antibiotic treatment. No microscopic differences were observed in the animals receiving FMTs (day 32 and 46; Fig. 4B).

\section{FMT does not affect gut permeability factors}

Gut permeability was indirectly evaluated by assessing the plasma levels of lipopolysaccharide binding protein (LBP; Fig. 5A) and also by measuring the mRNA expression of the tight junctions occludin and Zo1 in the colon (Fig. 5B and 5C, respectively). 
No significant alteration in the plasma levels of LBP was found among the experimental groups, a trend towards increase after the antibiotic treatment was observed on day7 (Fig. 5A). On the other hand, no difference was found in the plasma levels of LBP between CTR and DNBS donors (Fig. 5A).

RT-qPCR analysis of colon tight junctions revealed a marginal increase (not statistically significant) of occludin mRNA after the antibiotic treatment (day 7; Fig. 5B). On the contrary, on day 7, after the antibiotic regime, Zo-1 mRNA significantly increased (Fig. 5C). Neither occludin nor Zo-1 were affected by FMTs (Fig. $5 B$ and $5 C$, respectively).

\section{FMT alters gut cytokines profile, irrespective of the donor}

The expression of TNF- $a$, IL-6, IL-10 and TGF- $\beta$ mRNA level was measured in the colon by RT-qPCR. The antibiotic regimen caused a long-lasting up regulation of both pro- and anti-inflammatory cytokines expression (day 7; Fig. 6). This deregulatory effect of antibiotics did not change was not fixed by FMT (day 32; Fig. 6).

On the other hand, the cytokines profile of the FMT recipients did not match the pattern observed in the donors. The expression of both IL- 6 and TGF- $\beta$ resulted augmented in DNBS donors in respect to CTR donors but both FMT ${ }^{\mathrm{DNBS}}$ and FMT ${ }^{\mathrm{CTR}}$ recipient animals showed overexpression (day 32, Fig. 6B and 6D, respectively). Further, TNF-alpha and IL-10, unaltered in DNBS donors, were respectively down and upregulated in FMT ${ }^{\mathrm{DNBS}}$ recipients (day 32; Fig. $6 \mathrm{~A}$ and $6 \mathrm{~B}$, respectively). On day 46 all cytokines were still upregulated in the $a b x+$ vehicle group and in the $a b x+F M T^{C T R}$ group. By contrast, in the abx +FMTDNBS all cytokines, with exception of TGF- $\beta$, returned to the values observed in controls (Fig. 6).

\section{Transfer of microbiota from healthy controls counteracts post-inflammatory visceral pain persistence}

Finally, we tested the hypothesis that visceral pain could be suppressed or reduced by FMT from healthy donors. Pain threshold was assessed by evaluating the animal AWR in response to CRD stimulus (Fig. 7). As expected, 7 days after the induction of the damage, the abdominal withdrawal response to CRD was significantly higher in both the groups treated with DNBS. The FMT ${ }^{\mathrm{CTR}}$ led to a progressive reduction of visceral hypersensitivity in DNBS treated animals which became significantly lower after the third transplant cycle (day 28) and consolidated after the fourth one (day 35). The beneficial effect of FMT on post-inflammatory visceral pain caused by DNBS lasted up to 17 days after the treatment interruption (day 49). Starting from day 56 , the behavioural response of animals to CRD increase again, returning to the same value as the animals treated with DNBS + vehicle (Fig. 7).

\section{Discussion}

The present work highlights the relevance of microbiota in pain signalling from the gut. The FMT from animals affected by visceral pain to healthy rats was sufficient to induce visceral hypersensitivity. On the other hand, FMT from healthy donors induced a reduction of persistent visceral pain. A strong 
association between animals' pain threshold and microbiota composition changes was demonstrated. Indeed, intestinal inflammation as well as gut permeability and gut monoamines were found not to be involved in pain transfer mediated by FMT.

Although the gut microbiota appeared to be clearly involved in visceral pain regulation [3-5], the evidence collected so far are circumstantial [16-18]. Moreover, little is known on the evolution of microbiota over time after transplantation from animals affected by a disease, like pain, to naïve recipients. Here, we demonstrated a distinct temporal correlation between the animals' sensitivity and changes in the microbiota composition. As suggested by the analysis of the beta-diversity in samples from FMT ${ }^{\text {DNBS }}$ animals, major modifications in the composition and structure of the gut microbiota occurred after the FMT set II (Day 32), correlating with a significantly increased response of these animals to CRD. At a later stage (Day 46), when the difference in visceral sensitivity perception disappeared, a less pronounced variation in beta-diversity was observed.

Evaluation of taxonomic profiles confirmed that marked differences between the animals that underwent the FMT ${ }^{\mathrm{CTR}}$ or FMT ${ }^{\mathrm{DNBS}}$ were identifiable on Day 32 at phylum and family level for major members of gut microbiota, including protective microbial taxa known to be associated with gastrointestinal health (i.e. Akkermansiaceae) $[19,20]$. Akkermansia muciniphila is known to mediate a variety of host functions ranging from metabolism to immune regulation [21-23]. Regarding its role in pain syndromes or functional gastrointestinal disorders, $A$. muciniphila was found to be significantly reduced in children with IBS [24] as well as in animal models of post-inflammatory IBS [25]. Recently, Guida et al. reported that pain related to dysbiosis in vitamin $D$ deficient mice were ameliorated by an anandamide congener, in concomitance with increased levels of Akkermansia, Eubacterium and Enterobacteriaceae [26]. However, there is little evidence for a pain-alleviating effect mediated by A. muciniphila. One hypothesis is that SCFAs, like propionate and acetate, both end-products of mucus degradation by Akkermansia, may modulate visceral nociception, as stimulation of the SCFA receptor GPR43 has been shown to decrease visceral pain sensitivity in healthy humans $[27,28]$. The SCFAs have been associated to human gastrointestinal disorders [29] and proposed as regulators of visceral sensitivity since the activation of their receptors, FFAR2/3 regulates leucocyte functions, such as the production of cytokines (e.g. TNF-a, IL-2, IL-6, and IL-10), eicosanoids and chemokines (e.g. CCL2)[30]. Nevertheless, the role of SCFAs in visceral and somatic pain perception is still the subject of debate: depending on the context the effect of SCFAs signalling can be either analgesic or nociceptive [31]. Clinical evidence has shown that butyrate, a SCFAs found dramatically reduced in the faecal samples of IBS and IBD patients, is highly effective in relieving abdominal pain $[32,33]$. The significant reduction of butyrate in the FMT ${ }^{\text {DNBS }}$ group on Day 32 is in line with this evidence. Nevertheless, despite the reduction of butyrate on Day 32, the total amount of SCFAs increased in these animals both on Day 18 and 32 mainly contributed by an overproduction of acetate. Specific bacteria taxa were found to be negatively correlated with the increased acetate levels uniquely on Day 32, suggesting the presence of a defined microbiota profile at this stage. Of note, the recently identified bacterial genus Acetatifactor belonging to Lachnospiraceae, one of the most prominent bacterial family being expanded in samples from the FMT ${ }^{\mathrm{DNBS}}$ group, and showing strong acetate- 
producing capabilities [34], resulted overrepresented in FMT ${ }^{\mathrm{DNBS}}$ samples (Figure S4B). However, there are no consistent data in literature reporting a negative impact of acetate on gut physiology.

By monitoring the time course modifications and evolution of the gut microbiota in this model, emerged that the effects of FMT were long-lasting but temporary, demonstrating the presence of a continuous bidirectional influence between the microbiota and the gut, as previously postulated $[35,36]$. FMT ${ }^{\mathrm{DNBS}}$ induced visceral hypersensitivity was evident already $24 \mathrm{~h}$ after the first set of FMT, suggesting the presence of pain mediators in the supernatant obtained from the faeces, as supposed in other studies $[17,37]$. The presence of pain mediators in the faecal medium might explain the acute effect of FMT. By contrast, the long-lasting hyperalgesia observed in these animals is not attributable to the acute stimulation, nor to local intestinal irritation, since colon histological analysis revealed no damage on Day 32 ( 7 days after the last FMT). These findings support the idea of an active role of microbiota in pain induction mediated by the FMT. Besides, the apparent longer persistence of visceral hypersensitivity in the AWR test in respect to VMR test, suggested a possible central sensitization to the painful stimulus induced by CRD. Similarly, in the animals treated with DNBS, VMR response was significantly decreased after 4 weeks, while the augmented AWR response persisted up to 13 weeks after DNBS injection [38].

A slight increase in gut permeability occurred after the antibiotic treatment, with an increase in the expression of Zo-1, which has been positively correlated with a leaky gut [39], confirming the positive effect of microbiota on gut barrier integrity maintenance [40].

The bacterial community participates in maintaining intestinal homeostasis through the "training" of the immune system and inhibiting growth of pathogens [41]. The analysis of gene expression in the gut confirmed a derangement of cytokines as a result of both the antibiotic treatment and the FMT, but no significant correlations were found between the cytokines profiles among the groups and the observed differences in visceral pain.

Then we analysed the effect of FMT on tryptophan metabolism (Table S1-2), considering its relevance in the microbiota-gut-brain axis as well as in the pathophysiology of both IBS and IBDs [42]. A decrease in plasma tryptophan was found in DNBS donors, likely consequent to an increased biosynthesis of serotonin in the gut, as confirmed by the HPLC (Table S3-4). This phenomenon, which is a characteristic consequence of intestinal damages, can directly influence the neuronal firing of visceral afferents [ 43 , 44]. A plasma tryptophan decrease was also detected in the animals underwent FMT and correlated to a massive and long-lasting increase of serotonin in the faeces (Table S6), but not in the gut tissue (Table S3). An increase of $5-\mathrm{HT}$ in the gut lumen has been recently demonstrated to selectively modulate the colonization of bacteria species in the gut [45]. Nevertheless, no difference was found between the animals receiving the FMT from CTR or from DNBS donors, demonstrating again that $5-\mathrm{HT}$ is not involved in the transfer of pain mediated by FMT ${ }^{\mathrm{DNBS}}$.

The decrease of dopamine consequent to the antibiotic treatment and the FMT (Table S5 and S7) resulted in accordance to previous data reporting an antibiotic-mediated reduction of this 
neurotransmitter in the gut, where it plays a role in gastrointestinal motility regulation [46].

The anti-hyperalgesic effect obtained by transplanting a healthy microbiota in DNBS animals contributes further to sustain the role of microbiota in pain signalling. Currently, many discrepancies do exist on the therapeutic potential of FMT [47]. So far it has been considered a good protocol to maintain a low number of FMT and supplied by a single donor $[48,49]$. However, recent clinical studies highlighted the efficacy of a multiple donor and repeated treatment-based therapy in patients affected by UC, chronic intestinal pseudo-obstruction and IBS [50-52]. In certain pathological conditions, the interactions between genetic, immunological, and environmental factors might determine a state more resistant to therapeutic microbial manipulation [53]. In the preclinical model of colitis-induced visceral hypersensitivity, we obtained a substantial reduction in pain after 4 cycles of FMT, utilizing different donors. It is likely that the "inflammatory" environment wherein the microbiota is transplanted may pose as an obstacle to the engraftment of some species of microbiota compared to others. In this case, the repetition of the FMT could "force" the host to accept the newly introduced microbiota. These findings suggest the possibility to enhance the therapeutic efficacy of FMT by developing different protocols according to the specific disorder to be treated.

\section{Conclusions}

The results obtained in vivo strengthen the hypothesis of an active contribution of gut microbiota to visceral pain induction and persistence and encourage the continued investigation into the mechanisms by which the microbiota modulates visceral sensitivity, in order to make it a suitable target for the treatment of abdominal pain.

\section{Methods}

\section{Animals}

For all the experiments described below, Male Sprague-Dawley rats (Envigo, Varese, Italy) weighing approximately $220-250 \mathrm{~g}$ at the beginning of the experimental procedure, were used. Animals were housed in CeSAL (Centro Stabulazione Animali da Laboratorio, University of Florence) and used at least 1 week after their arrival. Four rats were housed per cage (size $26 \times 41 \mathrm{~cm})$; animals were fed a standard laboratory diet and tap water ad libitum, and kept at $23 \pm 1{ }^{\circ} \mathrm{C}$ with a $12 \mathrm{~h} \mathrm{light/dark} \mathrm{cycle,} \mathrm{light} \mathrm{at} 7$ a.m. All animal manipulations were carried out according to the Directive 2010/63/EU of the European parliament and of the European Union council (22 September 2010) on the protection of animals used for scientific purposes. The ethical policy of the University of Florence complies with the Guide for the Care and Use of Laboratory Animals of the US National Institutes of Health (NIH Publication No. 85 - 23, revised 1996; University of Florence assurance number: A5278-01). Formal approval to conduct the described experiments was obtained from the Animal Subjects Review Board of the University of Florence. Experiments involving animals have been reported according to ARRIVE guidelines [54]. All efforts were made to minimize animal suffering and to reduce the number of animals used. 


\section{Induction of colitis}

Colitis was induced in accordance with the method described previously by Antonioli et al. [55] with minor changes. In brief, during a short anaesthesia with isoflurane (2\%), $30 \mathrm{mg}$ of 2,4-dinitrobenzenesulfonic acid (DNBS) in $0.25 \mathrm{ml}$ of $50 \%$ ethanol was administered intrarectally via a polyethylene PE- 60 catheter inserted $8 \mathrm{~cm}$ proximal to the anus. Control rats received $0.25 \mathrm{ml}$ of saline solution.

\section{Faecal Microbiota Transplantation (FMT) study design}

In the first experimental set, rats were randomized into the following treatment groups $(n=10)$ : control (vehicle, no antibiotic treatment, no FMT); abx + vehicle (antibiotic treatment followed by vehicle administration); $a b x+$ FMT ${ }^{\text {CTR }}$ (antibiotic treatment followed by FMT from controls); abx + FMTDNBS (antibiotic treatment followed by FMT from DNBS treated animals). The animals were singly housed and underwent the following antibiotic/antifungal regimen to prepare them to the FMT: day $0-6$ rats received a daily oral gavage of amphotericin $B\left(1 \mathrm{mg} \mathrm{kg}^{-1}\right)$ and metronidazole $\left(100 \mathrm{mg} \mathrm{kg}^{-1}\right)$ while an antibiotic mix (ceftazidime $1 \mathrm{~g} \mathrm{~L}^{-1}$, vancomycin $0.5 \mathrm{~g} \mathrm{~L}^{-1}$ and neomicin $1 \mathrm{~g} \mathrm{~L}^{-1}$ ) was added to the autoclaved drinking water and changed every two days. On day 7,24 h after the interruption of the antibiotic treatment, the animals underwent the FMT procedure. FMT was daily performed on days 7-11 (Set I) and on days 20-24 (Set II). Behavioural tests were performed at the end of the antibiotic treatment (before starting the FMT), $24 \mathrm{~h}$ and 7 days after each FMT set and once week after the last treatment. Effects of the antibiotic/antifungal treatments on the gut microbiota were evaluated by a culture-based analysis for each treatment group. A portion of fresh faecal pellets $(50 \mathrm{mg})$ was dissolved in $1 \mathrm{ml}$ of sterile saline by vigorous vortexing, and the mean viable cell count was then determined by plating $0.1 \mathrm{ml}$ of appropriate dilutions (detection range, $2 \times 10^{2}$ to $16 \times 10^{8} \mathrm{CFU} \backslash \mathrm{g}$ of faecal pellet) onto: i) MacConkey agar (Oxoid, Cheshire, UK), selective for isolation of Gram-negative enteric bacteria; ii) CNA-CV Agar (Becton, Dickinson and Company, Franklin Lakes, NJ, USA), selective for isolation of Gram-positive cocci; and iii) Sabouraud glucose agar (Becton, Dickinson and Company, Franklin Lakes, NJ, USA), selective for isolation of yeasts. For the FMT, faecal material was collected from controls $(n=3)$ and DNBS treated animals $(n=3)$ (between 14 and 21 days after the intra-rectal injection of the inflammatory agent) and placed into tubes containing sterile saline solution, tubes were left on ice for $60 \mathrm{~min}$ and later homogenized for $2 \mathrm{~min}$ on ice using a hand-held pellet pestle device with sterile, re-usable pestles. When fully homogenized, the suspended pellets were directly utilized for the FMT procedure. FMT was carried out via oral gavage with a faecal suspension $\left(50 \mathrm{mg} \mathrm{ml}^{-1}\right.$ ) in a final volume of $3 \mathrm{ml}$.

In the second experimental set, rats were randomized into the following treatment groups $(n=5)$ : control (vehicle); DNBS + vehicle (intrarectal injection of DNBS $30 \mathrm{mg}$ followed by vehicle administration); DNBS + FMT $^{\text {CTR }}$ (intrarectal injection of DNBS $30 \mathrm{mg}$ followed by FMT from controls). Colitis was induced in the animals by the intra rectal injection of DNBS (30 mg in $0.25 \mathrm{~mL} \mathrm{EtOH} 50 \%$ ) on day 1 . A control group was intra-rectally administered with saline solution. Seven day after DNBS injection the animals were split into 2 groups, respectively receiving the vehicle or the naïve controls-derived faecal microbiota suspensions (FMT ${ }^{\text {CTR }}$ ). FMT was performed for 5 consecutive days/week and the same protocol was repeated for 4 
weeks (IIV set of FMT): on days 7-11 (Set I), 14-18 (Set II), 21-25 (Set III) and 28-32 (Set IV) after DNBS injection. Behavioural tests were carried out 7 days after DNBS injection (before starting FMT), 3 days after each FMT set and once week after the last treatment. For the FMT, faecal material was collected from control naïve animals $(n=3)$ and placed into tubes containing sterile saline solution, tubes were left on ice for $60 \mathrm{~min}$ and later homogenized for two min on ice using a hand-held pellet pestle device with sterile, re-usable pestles. When fully homogenized, the suspended pellets were directly utilized for the FMT procedure. FMT was carried out via oral gavage with a faecal suspension $\left(50 \mathrm{mg} \mathrm{ml}^{-1}\right)$ in a final volume of $3 \mathrm{ml}$.

\section{Assessment of visceral sensitivity by Viscero Moto Response (VMR)}

The visceromotor response (VMR) to colorectal balloon distension were used as objective measure of visceral sensitivity in animals. Two EMG electrodes were sutured into the external oblique abdominal muscle under deep anaesthesia and exteriorised dorsally [56]. VMR assessment were carried out under light anaesthesia (isoflurane 2\%) as previously reported [57]. A lubricated latex balloon (length: $4.5 \mathrm{~cm}$ ), assembled to an embolectomy catheter and connected to a syringe filled with water was used to perform colo-rectal distension. The balloon was inserted into the colon and positioned $6.5 \mathrm{~cm}$ from the anus and was filled with increasing volumes of water $(0.5,1,2,3 \mathrm{~mL})$. The electrodes were relayed to a data acquisition system and the corresponding EMG signal consequent to colo-rectal stimulation were recorded, amplified and filtered (Animal Bio Amp, ADInstruments, USA), digitised (PowerLab 4/35, ADlinstruments, USA), analysed and quantified using LabChart 8 (ADInstruments, USA). To quantify the magnitude of the VMR at each distension volume, the area under the curve (AUC) immediately before the distension (30 s) was subtracted from the AUC during the balloon distension (30 s) and responses were expressed as percentage increase from the baseline. The time elapsed between two consecutive distension was 5 min.

\section{Assessment of visceral sensitivity by Abdominal Withdrawal Reflex (AWR)}

The behavioural responses to CRD were assessed in the animals by measuring the Abdominal Withdrawal Reflex (AWR), a semi-quantitative score described previously in conscious animals [58]. Briefly, rats were anesthetized with isoflurane, and a lubricated latex balloon (length: $4.5 \mathrm{~cm}$ ), attached to polyethylene tubing, assembled to an embolectomy catheter and connected to a syringe filled with water was inserted through the anus into the rectum and descending colon of adult rats. The tubing was taped to the tail to hold the balloon in place. Then rats were allowed to recover from the anaesthesia for $30 \mathrm{~min}$. AWR measurement consisted of visual observation of animal responses to graded $C R D(0.5,1,2,3 \mathrm{~mL})$ blinded observers who assigned AWR scores: No behavioral response to colorectal distention ( 0 ); Immobile during colorectal distention and occasional head clinching at stimulus onset (1); Mild contraction of the abdominal muscles but absence of abdomen lifting from the platform (2); Observed strong contraction of the abdominal muscles and lifting of the abdomen off the platform (3); Arching of 
the body and lifting of the pelvic structures and scrotum (4). The time elapsed between two consecutive distension was 5 min.

\section{Profiling of gut microbiota}

Fecal pellets $(50 \mathrm{mg}$ ) and fecal suspensions (volume equivalent to $50 \mathrm{mg}$ ) were processed for the total DNA extraction using the DNeasy PowerLyzer PowerSoil Kit (Qiagen, Hilden, Germany). Next generation sequencing of $16 \mathrm{~S}$ ribosomal RNA amplicons (V3-V4 regions) was performed using the Illumina MiSeq platform, according to the Illumina 16S Metagenomic Sequencing Library Preparation protocol (Part \# 15044223 Rev. B; URL: http://www.illumina.com/content/dam/illumina-

support/documents/documentation/chemistry_documentation/16s/16s-metagenomic-library-prep-guide15044223-b.pdf), following a $2 \times 300$ bp paired-end approach. Sequencing results were analyzed using the QIIME 2 suite (Quantitative Insights Into Microbial Ecology)[59]. Briefly, following raw reads denoising (i.e. error correction, removal of chimeric and singleton sequences, join of denoised paired-end reads) using DADA2 [60] for each sample amplicon sequence variants (ASVs) were inferred. Taxonomic classification of dereplicated ASV was performed using a Naive Bayes classifier trained on the SILVA 16S reference database (release 132) (https://www.arb-silva.de/documentation/release-132/). Microbial diversity was estimated by evaluating alpha-diversity (Shannon index) and beta-diversity (Bray-Curtis) metrics using specific tools implemented in the QIIME 2 pipeline. The 16S rRNA sequence data has been deposited in the NCBI Sequence Read Archive (SRA) under the BioProject accession number PRJNA605112.

The gut microbiota was characterized in representative animals, at different sampling points (Day 18,32 , $46)$, belonging to the following treatment groups: vehicle + vehicle $(n=3)$; $a b x+$ vehicle $(n=4)$; $a b x+$ $\operatorname{FMT}^{\text {CTR }}(n=4) ; a b x+$ FMT $^{\text {DNBS }}(n=4)$. The first and the last faecal suspension utilized in the I and II set of FMT, prepared with faecal material from controls and DNBS-treated animals, were also analysed $(n=8)$.

\section{Gas Chromatography Mass Spectrometry (GC-MS) Analysis of SCFAs}

Methanol and tert-butyl methyl ether (Chromasolv grade), sodium bicarbonate and hydrochloric acid (reagent grade), (2H3)Acetic, (2H3)Propionic, (2H7)iso-Butyric and (2H9)iso-Valeric (used as internal standards (ISTDs)), acetic acid, propionic acid, butyric acid, isobutyric acid, valeric acid, and isovaleric acid (analytical standards grade) were purchased by Sigma-Aldrich (Milan, Italy). MilliQ water $18 \mathrm{MW}$ was obtained from Millipore's Simplicity system (Milan, Italy). The analysis of short-chain fatty acids (SCFAs) was performed by an Agilent GC-MS system composed with a 5971 single quadrupole mass spectrometer, 5890 gas-chromatograph, and 7673 autosampler.

The SCFAs in the fecal samples were analyzed as free acid form using a SupelcoNukol column, with a $30 \mathrm{~m}$ length, $0.25 \mathrm{~mm}$ internal diameter, and $0.25 \mu \mathrm{m}$ of film thickness with the temperatures program as follows: Initial temperature of $40^{\circ} \mathrm{C}$ was held for $1 \mathrm{~min}$, then it was increased to $150^{\circ} \mathrm{C}$ at $30{ }^{\circ} \mathrm{C} / \mathrm{min}$, and finally increased to $220^{\circ} \mathrm{C}$ at $20^{\circ} \mathrm{C} / \mathrm{min}$. A $1 \mu \mathrm{L}$ aliquot of the extracted sample was injected in splitless 
mode (splitless time $1 \mathrm{~min}$ ) at $250^{\circ} \mathrm{C}$, while the transfer line temperature was $280^{\circ} \mathrm{C}$. The carrier flow rate was maintained at $1 \mathrm{~mL} / \mathrm{min}$. The quantitative determination of SCFAs in each sample was carried out by the ratio between the area abundance of the analytes with the area abundance of respective labeled internal standard (isotopic dilution method). The value of this ratio was named Peak Area Ratio (PAR) and it was used as abundance of each analyte in the quantitative evaluation. The ionic signals of SCFAs' and the reference internal standards used for the quantitation of each SCFAs were reported in the Supplementary information. Faecal samples were added to sodium bicarbonate $10 \mathrm{mM}$ solution (1:1 $\mathrm{w} / \mathrm{v})$. The mixture was sonicated $5 \mathrm{~min}$ and centrifuged at $5000 \mathrm{rpm}$ for $10 \mathrm{~min}$. The supernatants were frozen at $-20^{\circ} \mathrm{C}$ until analysis. The SCFAs were finally extracted as follows: an aliquot of $100 \mu \mathrm{l}$ of sample solution (corresponding to $0.1 \mathrm{mg}$ of stool sample) was added of $50 \mu \mathrm{L}$ of ISTDs mixture, $1 \mathrm{~mL}$ of tert-butyl methyl ether and $50 \mu \mathrm{L}$ of $1.0 \mathrm{M} \mathrm{HCl}$ solution in $1.5 \mathrm{~mL}$ centrifuge tube. Afterwards, each tube was shaken in vortex apparatus for 2 minutes, centrifuged at $10000 \mathrm{rpm}$ for 5 minutes, and finally the solvent layer was transferred in autosampler vial and analyzed by GC-MS method. Each sample was prepared and processed, by the method described above in triplicate. Further details are reported in the Supplementary information.

\section{Histological evaluation of colonic damage}

The evaluation of colon damage was performed both macroscopically and histologically, in accordance with the criteria previously reported by Antonioli et al. [55]. The macroscopic criteria were: presence of adhesions between colon and other intra-abdominal organs ( $0-2)$; consistency of colonic faecal material (indirect marker of diarrhoea; 0-2); thickening of colonic wall ( $\mathrm{mm})$; presence and extension of hyperaemia and macroscopic mucosal damage (0-5). A microscopic analysis was carried out on haematoxylin/eosin-stained sections of formalin-fixed full-thickness samples obtained from the distal colon. The microscopic damage was assessed in accordance with the criteria reported previously by Antonioli et al. [61] by evaluating the presence of mucosal architecture loss, goblet cell depletion, crypt abscess, cellular infiltration, tunica muscularis thickening $(n=4-6)$.

\section{Plasma Lipopolysaccharide Binding Protein (LBP) by ELISA}

Plasma LBP concentrations were determined using the Enzyme Immunoassay Kit (Enzo®, Life Sciences). Rat blood samples were collected in heparinized tubes after decapitation. Samples were centrifuged immediately at $14000 \mathrm{rpm}$ for 15 minutes and the resulting plasma was frozen at $-80^{\circ} \mathrm{C}$ until analysis. LBP concentrations were determined using the Enzyme Immunoassay Kit for free mouse and rat LBP as per manufacturers' instruction (Enzo®, Life Sciences). Sensitivity: Range $1-50 \mathrm{ng} / \mathrm{ml}(\mathrm{n}=3-5)$.

\section{Gene expression analysis by quantitative RT-PCR (qRT-PCR)}

Total RNA was extracted from colon samples using the mirVana ${ }^{\text {TM }}$ miRNA Isolation kit (Ambion/life technologies) according to the manufacturer's recommendations. RNA concentration and quality were determined using a Nanodrop 1000 (Thermo Scientific). Quantitative PCR was carried out in a LightCycler480 System using PowerUp ${ }^{\text {TM }}$ SYBR ${ }^{\circledR}$ Green Master Mix (Applied Biosystems) and specific probes designed by Applied Biosystems to rat occludin, ZO-1, TNF-a, IL6, IL10 and Tgf- $\beta$, while using $\beta$ - 
Actin as an endogenous control. Experimental samples were run in triplicate with $4 \mu \mathrm{L}\left(10 \mathrm{ng} \mathrm{L}^{-1}\right) \mathrm{cDNA}$ per reaction. To check for amplicon contamination, each run contained no template controls in triplicate for each probe used. Cycle threshold (Ct) values were recorded. Data was normalised using $\beta$-Actin and transformed using the $2^{-\Delta \Delta C t}$ method. No significant differences were observed in the mRNA expression levels of $\beta$-Actin between groups $(n=4-6)$.

\section{Statistical analysis}

All the experimental procedures were performed by a researcher blind to the treatment. Results were expressed as mean \pm S.E.M. The analysis of variance of behavioural data was performed by one-way ANOVA with Bonferroni's significant difference procedure used for post-hoc comparisons. Statistics of electrophysiological data was performed by Student's paired or unpaired t-test or by one-way ANOVA followed by Bonferroni's post-test, as appropriate. $P$ values of less than 0.05 were considered significant. Data were analyzed using the "Origin 9" software (OriginLab, Northampton, USA). Statistical analysis on 16S sequencing data was performed by QIIME2 using nonparametric tests. Differences in relative abundance of bacterial taxa were evaluated using the Kruskal-Wallis test on pairwise or multiple comparisons. The permutational ANOVA (PERMANOVA) test was applied to beta-diversity distance matrices generated by QIIME2 to test significance between samples' clusters observed following Principal Coordinate Analysis (PCoA); significance was determined through 999 permutations. Spearman correlation coefficients between relative abundances of microbial taxa and levels of SCFAs were computed using GraphPad Prism 6 (GraphPad Software, La Jolla, CA).

\section{Declarations}

\section{Acknowledgments}

We are grateful to Dr G. Clarke (APC Microbiome Ireland \& Department of Psychiatry and Neurobehavioural Science, University College Cork, Cork, Ireland) and Dr K. Rea (APC Microbiome Ireland, University College Cork, Cork, Ireland) for their practical and theoretical input in the work.

\section{Author contributions}

E.L., L.D.C.M, C.N., V.D.P. and S.O.M. conceived and designed the study. E.L., C.P., E.N., S.B., G.B. performed the experiments. L.M., A.T. analysed the data. E.L., L.D.C.M., V.D.P. wrote the manuscript. A.P., C.N., A.A. and S.O.M. revised the manuscript. C.G., G.M.R. A.A. and J.F.C. supervised the study and provided the experimental resources.

\section{Funding}

This research was supported by the Italian Ministry of Instruction, University and Research, by the University of Florence and by the University College Cork.

\section{Availability of data and materials}


The datasets generated during and analysed during the current study are available from the corresponding author on reasonable request.

\section{Competing interests}

The authors declare that they have no competing interest.

\section{Ethics approval and consent to participate}

All experiments were performed using rodents in accordance with the International Association for the Study of Pain, the European Union directives and the National Institutes of Health guidelines on laboratory animal welfare and approved by the Animal Care committees at Universities of Florence (Italy) and Cork (Ireland).

\section{Consent for publication}

Not applicable.

\section{References}

1. Camilleri M, Boeckxstaens G. Dietary and pharmacological treatment of abdominal pain in IBS. Gut. 2017;66:966-974.

2. Stern EK, Brenner DM. Gut Microbiota-Based Therapies for Irritable Bowel Syndrome. Clin Transl Gastroenterol. 2018;9:e134.

3. Hyland NP, Quigley EMM, Brint E. Microbiota-host interactions in irritable bowel syndrome: epithelial barrier, immune regulation and brain-gut interactions. World J Gastroenterol. 2014;20:8859-8866.

4. O'Mahony SM, Clarke G, Dinan TG, Cryan JF. Early-life adversity and brain development: Is the microbiome a missing piece of the puzzle? Neuroscience. 2017;342:37-54.

5. Foster JA, Rinaman L, Cryan JF. Stress \& the gut-brain axis: Regulation by the microbiome. Neurobiol Stress. 2017;7:124-136.

6. Klem F, Wadhwa A, Prokop LJ, Sundt WJ, Farrugia G, Camilleri M, Singh S, Grover M. Prevalence, Risk Factors, and Outcomes of Irritable Bowel Syndrome After Infectious Enteritis: A Systematic Review and Meta-analysis. Gastroenterology. 2017;152:1042-1054.e1041.

7. O'Mahony SM, Felice VD, Nally K, Savignac HM, Claesson MJ, Scully P, Woznicki J, Hyland NP, Shanahan F, Quigley EM, et al. Disturbance of the gut microbiota in early-life selectively affects visceral pain in adulthood without impacting cognitive or anxiety-related behaviors in male rats. Neuroscience. 2014;277:885-901.

8. Verdú EF, Bercik P, Verma-Gandhu M, Huang XX, Blennerhassett P, Jackson W, Mao Y, Wang L, Rochat F, Collins SM. Specific probiotic therapy attenuates antibiotic induced visceral hypersensitivity in mice. Gut. 2006;55:182-190. 
9. Hoban AE, Moloney RD, Golubeva AV, McVey Neufeld KA, O'Sullivan O, Patterson E, Stanton C, Dinan TG, Clarke G, Cryan JF. Behavioural and neurochemical consequences of chronic gut microbiota depletion during adulthood in the rat. Neuroscience. 2016;339:463-477.

10. Luczynski P, Tramullas M, Viola M, Shanahan F, Clarke G, O'Mahony S, Dinan TG, Cryan JF. Microbiota regulates visceral pain in the mouse. Elife. 2017;6: 6:e25887.

11. Fung TC. The microbiota-immune axis as a central mediator of gut-brain communication. Neurobiol Dis. 2020;136:104714.

12. Rhee SH, Pothoulakis $\mathrm{C}$, Mayer EA. Principles and clinical implications of the brain-gut-enteric microbiota axis. Nat Rev Gastroenterol Hepatol. 2009;6:306.

13. Cryan JF, Dinan TG. Mind-altering microorganisms: the impact of the gut microbiota on brain and behaviour. Nat Rev Neurosci. 2012;13:701-712.

14. Mayer EA, Tillisch K, Gupta A. Gut/brain axis and the microbiota. J Clin Invest 2015, 125:926-938.

15. Franzosa EA, Sirota-Madi A, Avila-Pacheco J, Fornelos N, Haiser HJ, Reinker S, Vatanen T, Hall AB, Mallick $\mathrm{H}$, Mclver LJ, et al. Gut microbiome structure and metabolic activity in inflammatory bowel disease. Nat Microbiol. 2019;4:293-305.

16. Crouzet L, Gaultier E, Del'Homme C, Cartier C, Delmas E, Dapoigny M, Fioramonti J, BernalierDonadille A. The hypersensitivity to colonic distension of IBS patients can be transferred to rats through their fecal microbiota. Neurogastroenterol Motil. 2013;25:e272-282.

17. Wang P, Du C, Chen F-X, Li C-Q, Yu Y-B, Han T, Akhtar S, Zuo X-L, Tan X-D, Li Y-Q. BDNF contributes to IBS-like colonic hypersensitivity via activating the enteroglia-nerve unit. Sci Rep. 2016;6:20320.

18. Hadizadeh F, Bonfiglio F, Belheouane M, Vallier M, Sauer S, Bang C, Bujanda L, Andreasson A, Agreus $L$, Engstrand $L$, et al. Faecal microbiota composition associates with abdominal pain in the general population. Gut. 2018;67:778-779.

19. Cani PD. Human gut microbiome: hopes, threats and promises. Gut. 2018;67:1716-1725.

20. Macchione IG, Lopetuso LR, laniro G, Napoli M, Gibiino G, Rizzatti G, Petito V, Gasbarrini A, Scaldaferri F. Akkermansia muciniphila: key player in metabolic and gastrointestinal disorders. Eur Rev Med Pharmacol Sci. 2019;23:8075-8083.

21. Dao MC, Everard A, Aron-Wisnewsky J, Sokolovska N, Prifti E, Verger EO, Kayser BD, Levenez F, Chilloux J, Hoyles L, et al. Akkermansia muciniphila and improved metabolic health during a dietary intervention in obesity: relationship with gut microbiome richness and ecology. Gut. 2016;65:426436.

22. Derrien M, Belzer C, de Vos WM. Akkermansia muciniphila and its role in regulating host functions. Microb Pathog. 2017;106:171-181.

23. Ouyang J, Lin J, Isnard S, Fombuena B, Peng X, Marette A, Routy B, Messaoudene M, Chen Y, Routy JP. The Bacterium Akkermansia muciniphila: A Sentinel for Gut Permeability and Its Relevance to HIV-Related Inflammation. Front Immunol. 2020;11:645. 
24. Rigsbee L, Agans R, Shankar V, Kenche H, Khamis HJ, Michail S, Paliy O. Quantitative profiling of gut microbiota of children with diarrhea-predominant irritable bowel syndrome. Am J Gastroenterol. 2012; 107:1740-1751.

25. Chen Y, Xiao S, Gong Z, Zhu X, Yang Q, Li Y, Gao S, Dong Y, Shi Z, Wang Y, et al. Wuji Wan Formula Ameliorates Diarrhea and Disordered Colonic Motility in Post-inflammation Irritable Bowel Syndrome Rats by Modulating the Gut Microbiota. Front Microbiol. 2017;8:2307-2307.

26. Guida F, Boccella S, Belardo C, lannotta M, Piscitelli F, De Filippis F, Paino S, Ricciardi F, Siniscalco D, Marabese I, et al. Altered gut microbiota and endocannabinoid system tone in vitamin $D$ deficiencymediated chronic pain. Brain Behav Immun. 2019;85:128-141.

27. Vanhoutvin SA, Troost FJ, Kilkens TO, Lindsey PJ, Hamer HM, Jonkers DM, Venema K, Brummer RJ. The effects of butyrate enemas on visceral perception in healthy volunteers. Neurogastroenterol Motil. 2009;21:952-e976.

28. Cruz-Aguliar RM, Wantia N, Clavel T, Vehreschild MJ, Buch T, Bajbouj M, Haller D, Busch D, Schmid RM, Stein-Thoeringer CK. An Open-Labeled Study on Fecal Microbiota Transfer in Irritable Bowel Syndrome patients reveals improvement in abdominal pain associated with the relative abundance of Akkermansia muciniphila. Digestion. 2019;100:127-138.

29. Niccolai E, Baldi S, Ricci F, Russo E, Nannini G, Menicatti M, Poli G, Taddei A, Bartolucci G, Calabrò AS, et al. Evaluation and comparison of short chain fatty acids composition in gut diseases. World $J$ Gastroenterol. 2019;25:5543-5558.

30. Vinolo MA, Rodrigues HG, Nachbar RT, Curi R. Regulation of inflammation by short chain fatty acids. Nutrients. 2011;3:858-876.

31. Kannampalli P, Shaker R, Sengupta JN. Colonic butyrate- algesic or analgesic? Neurogastroenterol Motil. 2011;23:975-979.

32. Huda-Faujan N, Abdulamir AS, Fatimah AB, Anas OM, Shuhaimi M, Yazid AM, Loong YY. The impact of the level of the intestinal short chain Fatty acids in inflammatory bowel disease patients versus healthy subjects. Open Biochem J. 2010;4:53-58.

33. Banasiewicz T, Krokowicz L, Stojcev Z, Kaczmarek BF, Kaczmarek E, Maik J, Marciniak R, Krokowicz $P$, Walkowiak J, Drews M. Microencapsulated sodium butyrate reduces the frequency of abdominal pain in patients with irritable bowel syndrome. Colorectal Dis. 2013;15:204-209.

34. Pfeiffer N, Desmarchelier C, Blaut M, Daniel H, Haller D, Clavel T. Acetatifactor muris gen. nov., sp. nov., a novel bacterium isolated from the intestine of an obese mouse. Arch Microbiol. 2012;194:901907.

35. Contijoch EJ, Britton GJ, Yang C, Mogno I, Li Z, Ng R, Llewellyn SR, Hira S, Johnson C, Rabinowitz $\mathrm{KM}$, et al. Gut microbiota density influences host physiology and is shaped by host and microbial factors. Elife. 2019;8:e40553.

36. Hevia A, Bernardo D, Montalvillo E, Al-Hassi HO, Fernandez-Salazar L, Garrote JA, Milani C, Ventura M, Arranz E, Knight SC, et al. Human colon-derived soluble factors modulate gut microbiota composition. Front Oncol. 2015;5:86. 
37. Buhner S, Li Q, Vignali S, Barbara G, De Giorgio R, Stanghellini V, Cremon C, Zeller F, Langer R, Daniel $\mathrm{H}$, et al. Activation of human enteric neurons by supernatants of colonic biopsy specimens from patients with irritable bowel syndrome. Gastroenterology. 2009;137:1425-1434.

38. Lucarini E, Parisio C, Branca JJV, Segnani C, Ippolito C, Pellegrini C, Antonioli L, Fornai M, Micheli L, Pacini A, et al. Deepening the Mechanisms of Visceral Pain Persistence: An Evaluation of the GutSpinal Cord Relationship. Cells. 2020;9.

39. Fasano A. Intestinal permeability and its regulation by zonulin: diagnostic and therapeutic implications. Clin Gastroenterol Hepatol. 2012;10:1096-1100.

40. Stevens BR, Goel R, Seungbum K, Richards EM, Holbert RC, Pepine CJ, Raizada MK. Increased human intestinal barrier permeability plasma biomarkers zonulin and FABP2 correlated with plasma LPS and altered gut microbiome in anxiety or depression. Gut. 2018;67:1555-1557.

41. Kamada N, Chen GY, Inohara N, Nunez G. Control of pathogens and pathobionts by the gut microbiota. Nat Immunol. 2013;14:685-690.

42. Agus A, Planchais J, Sokol H. Gut Microbiota Regulation of Tryptophan Metabolism in Health and Disease. Cell Host Microbe. 2018;23:716-724.

43. Mawe GM, Hoffman JM. Serotonin signalling in the gut-functions, dysfunctions and therapeutic targets. Nat Rev Gastroenterol Hepatol. 2013;10:473-486.

44. Nikolaus S, Schulte B, Al-Massad N, Thieme F, Schulte DM, Bethge J, Rehman A, Tran F, Aden K, Hasler R, et al. Increased Tryptophan Metabolism Is Associated With Activity of Inflammatory Bowel Diseases. Gastroenterology. 2017;153:1504-1516.e1502.

45. Fung TC, Vuong HE, Luna CDG, Pronovost GN, Aleksandrova AA, Riley NG, Vavilina A, McGinn J, Rendon T, Forrest LR, Hsiao EY. Intestinal serotonin and fluoxetine exposure modulate bacterial colonization in the gut. Nat Microbiol. 2019;4:2064-2073.

46. Li ZS, Schmauss C, Cuenca A, Ratcliffe E, Gershon MD. Physiological Modulation of Intestinal Motility by Enteric Dopaminergic Neurons and the $\mathrm{D}_{2}$ Receptor: Analysis of Dopamine Receptor Expression, Location, Development, and Function in Wild-Type and Knock-Out Mice. J Neurosci. 2006;26:2798.

47. Xu D, Chen VL, Steiner CA, Berinstein JA, Eswaran S, Waljee AK, Higgins PDR, Owyang C. Efficacy of Fecal Microbiota Transplantation in Irritable Bowel Syndrome: A Systematic Review and MetaAnalysis. Am J Gastroenterol. 2019;114:1043-1050.

48. Cammarota G, laniro G, Tilg H, Rajilic-Stojanovic M, Kump P, Satokari R, Sokol H, Arkkila P, Pintus C, Hart A, et al. European consensus conference on faecal microbiota transplantation in clinical practice. Gut .2017;66:569-580.

49. Wilson BC, Vatanen T, Cutfield WS, O'Sullivan JM. The Super-Donor Phenomenon in Fecal Microbiota Transplantation. Front Cell Infect Microbiol. 2019;9:2.

50. Paramsothy S, Kamm MA, Kaakoush NO, Walsh AJ, van den Bogaerde J, Samuel D, Leong RWL, Connor S, Ng W, Paramsothy R, et al. Multidonor intensive faecal microbiota transplantation for active ulcerative colitis: a randomised placebo-controlled trial. Lancet. 2017;389:1218-1228. 
51. Gu L, Ding C, Tian H, Yang B, Zhang X, Hua Y, Zhu Y, Gong J, Zhu W, Li J, Li N. Serial Frozen Fecal Microbiota Transplantation in the Treatment of Chronic Intestinal Pseudo-obstruction: A Preliminary Study. J Neurogastroenterol Motil. 2017;23:289-297.

52. El-Salhy M, Hausken T, Hatlebakk JG. Increasing the Dose and/or Repeating Faecal Microbiota Transplantation (FMT) Increases the Response in Patients with Irritable Bowel Syndrome (IBS). Nutrients. 2019;11:1415.

53. Sartor RB. Genetics and environmental interactions shape the intestinal microbiome to promote inflammatory bowel disease versus mucosal homeostasis. Gastroenterology. 2010;139:1816-1819.

54. McGrath JC, Lilley E. Implementing guidelines on reporting research using animals (ARRIVE etc.): new requirements for publication in BJP. Br J Pharmacol. 2015;172:3189-3193.

55. Antonioli L, Fornai M, Colucci R, Ghisu N, Da Settimo F, Natale G, Kastsiuchenka O, Duranti E, Virdis A, Vassalle $\mathrm{C}$, et al. Inhibition of adenosine deaminase attenuates inflammation in experimental colitis. J Pharmacol Exp Ther. 2007;322:435-442.

56. Christianson JA, Gebhart GF. Assessment of colon sensitivity by luminal distension in mice. Nat Protoc. 2007;2:2624-2631.

57. Parisio C, Lucarini E, Micheli L, Toti A, Di Cesare Mannelli L, Antonini G, Panizzi E, Maidecchi A, Giovagnoni E, Lucci J. Researching New Therapeutic Approaches for Abdominal Visceral Pain Treatment: Preclinical Effects of an Assembled System of Molecules of Vegetal Origin. Nutrients. 2020;12:22.

58. Chen Y, Lin C, Tang Y, Chen A-Q, Liu C-Y, Lu D-L. ZD 7288, an HCN channel blocker, attenuates chronic visceral pain in irritable bowel syndrome-like rats. World J Gastroenterol. 2014;20:2091-2097.

59. Bolyen E, Rideout JR, Dillon MR, Bokulich NA, Abnet CC, Al-Ghalith GA, Alexander H, Alm EJ, Arumugam M, Asnicar F, et al. Reproducible, interactive, scalable and extensible microbiome data science using QIIME 2. Nat Biotechnol. 2019;37:852-857.

60. Callahan BJ, McMurdie PJ, Rosen MJ, Han AW, Johnson AJA, Holmes SP: DADA2: High-resolution sample inference from Illumina amplicon data. Nat Methods. 2016;13:581-583.

61. Antonioli L, Fornai M, Colucci R, Awwad O, Ghisu N, Tuccori M, Del Tacca M, Blandizzi C. Differential recruitment of high affinity $\mathrm{A} 1$ and $\mathrm{A} 2 \mathrm{~A}$ adenosine receptors in the control of colonic neuromuscular function in experimental colitis. Eur J Pharmacol. 2011;650:639-649.

\section{Figures}




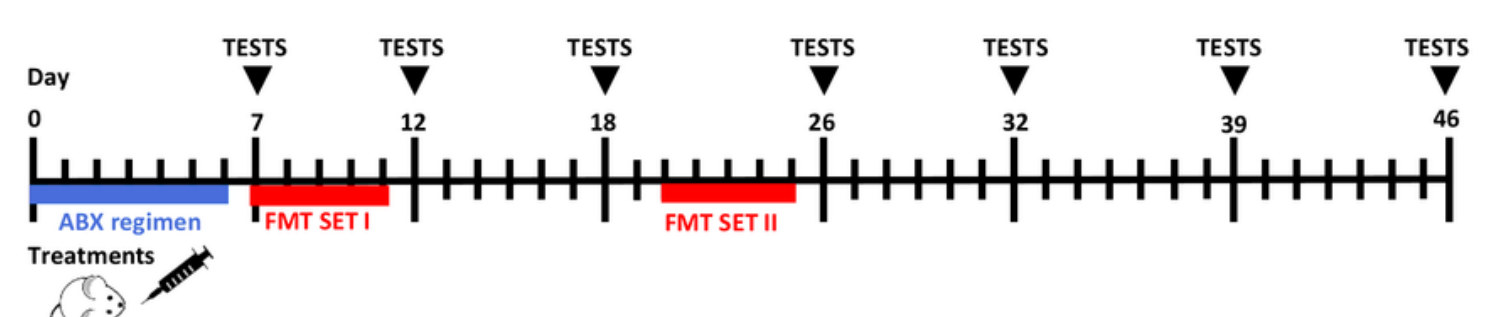

Day 7

before starting the FMT

A)



B)

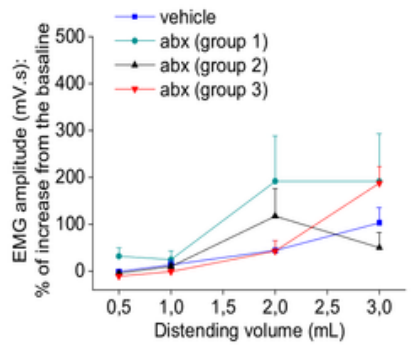

Day 12

24h after the FMT set I


Day 18

7 days after the FMT set I
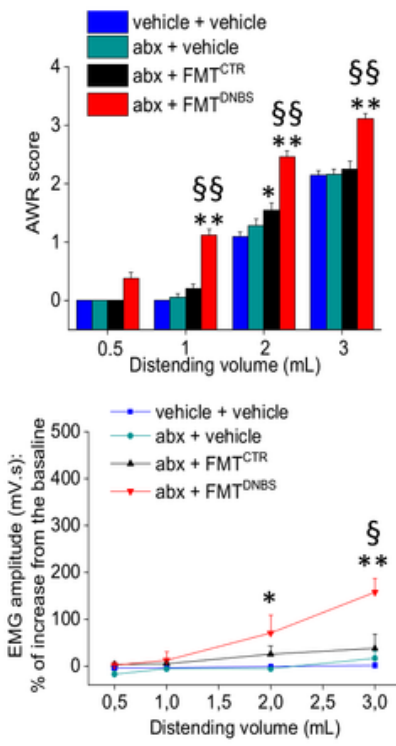

\section{Day 26}

24h after the FMT set II

Day 32

7 days after the FMT set II

Day 39

Day 46
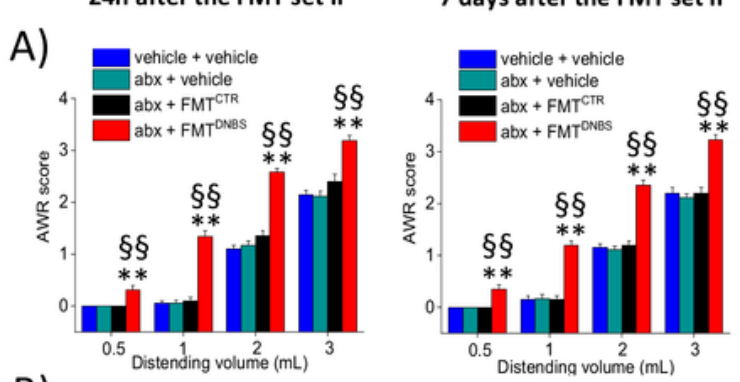

14 days after the FMT set II

21 days after the FMT set II

B)
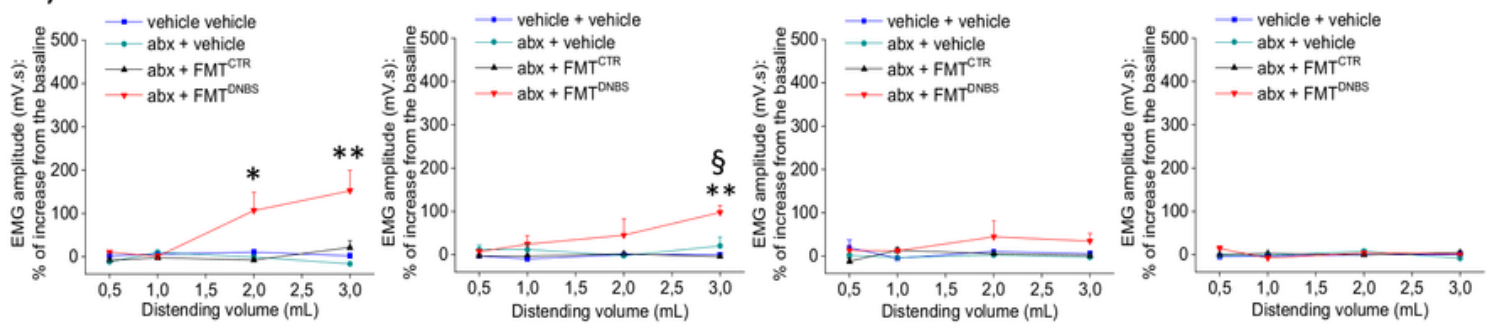

Figure 1

Effect of antibiotic treatment and FMT from DNBS-treated animals on visceral sensitivity of naïve recipients. As shown in the scheme, rats were treated, with a combination of antibiotics for 7 days; control group was treated with vehicle. On day 7 the antibiotics (abx)-treated animals were divided into 3 groups, FMT from CTR donors, FMT from DNBS donors or vehicle were respectively administered per os for 5 consecutive days. One week after the administrations were repeated. Behavioural tests were performed at 
the end of the antibiotic treatment, 24h and 7 days after each cycle of FMT and once week after the last treatment. Visceral sensitivity was assessed in the animals by measuring the Viscero-Motor Response (VMR; A) and the Abdominal Withdrawal Response (AWR; B) to Colo-Rectal Distension (CRD; 0.5-3 mL). Each value is the mean \pm S.E.M. of 5 (VMR) or 10 (AWR) rats per group. ${ }^{*} P<0.05$ and ${ }^{*} * \mathrm{P}<0.01$ vs vehicle or vehicle + vehicle treated animals. $\S \mathrm{P}<0.05$ and $\S \S \mathrm{P}<0.01$ vs abx + FMTCTR treated animals.

A)

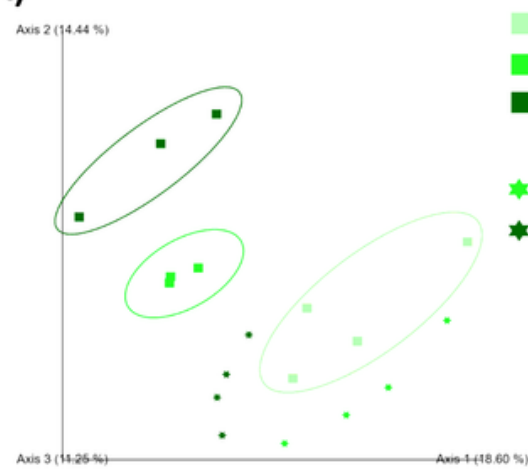

B)

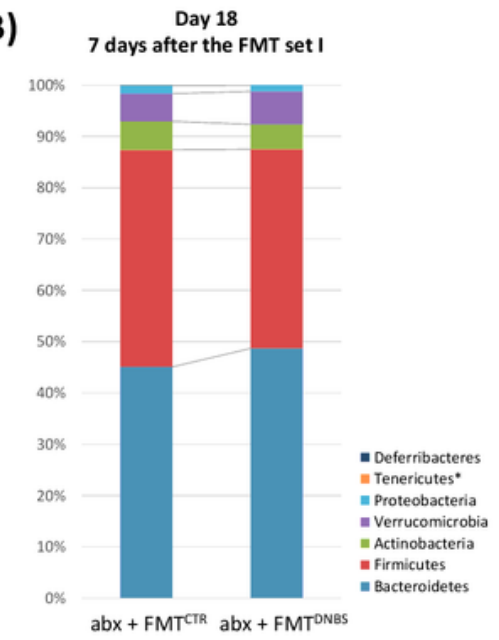

Day 18
C) 7 days after the FMT set I

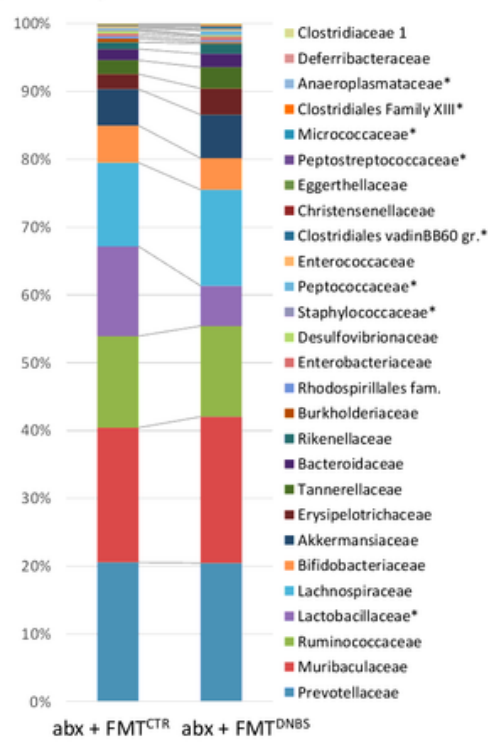

CTR (Day 0)

CTR (Day 18)

—CTR (Day 46)

* abx + vehicle (Day 18)

* abx + vehicle (Day 46)

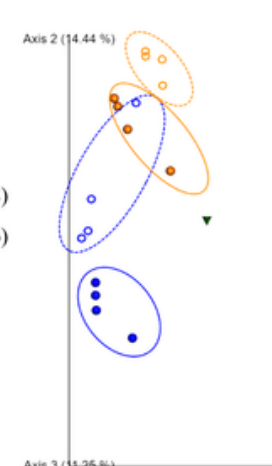

is 3

7 days after the FMT set II

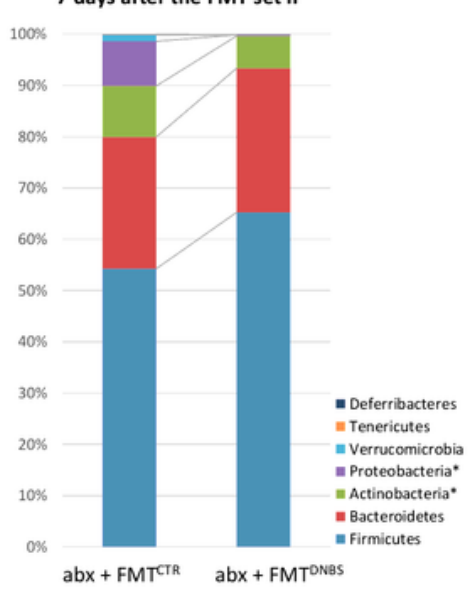

Day 32

7 days after the FMT set II

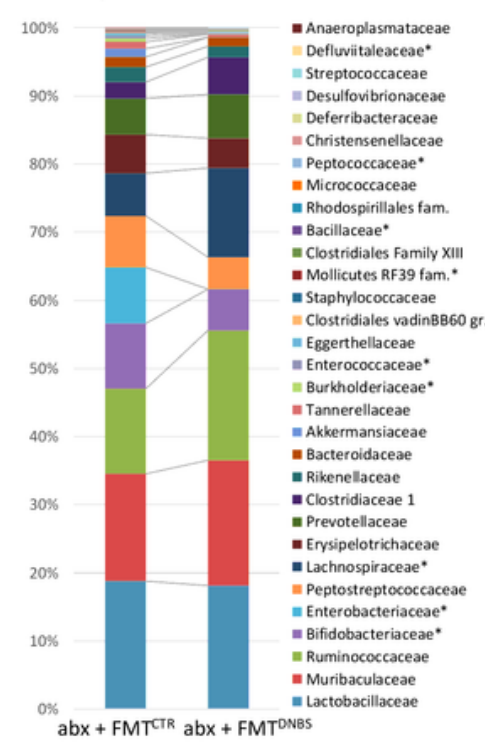

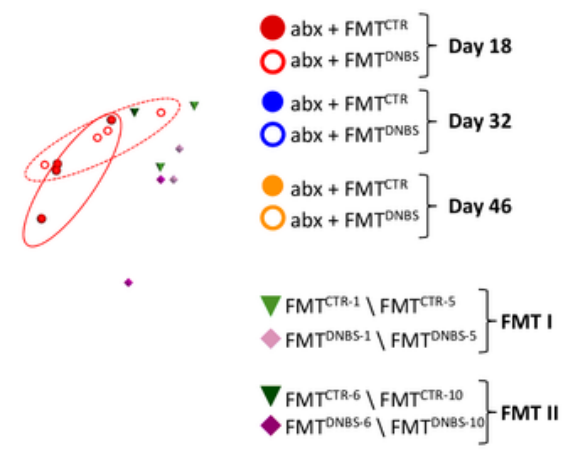

Oabx + FMT'DNBS - Day 18

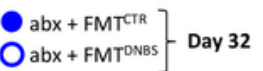

$\left.\begin{array}{c}a b x+F M T^{C T R} \\ a b x+F M T^{D N B S}\end{array}\right\}$ Day 46

$\left.\begin{array}{l}\nabla \text { FMT }^{\text {CTR-1 }} \backslash F \mathrm{MT}^{\text {CTR.5 }} \\ \mathrm{FMT}^{\text {DNBS-1 }} \backslash \mathrm{FMT}^{\text {ONBS-5 }}\end{array}\right]-$ FMT I

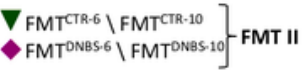


Analysis of beta-diversity and taxonomic profiles. A) PCoA plots based on the Bray-Curtis beta-diversity metric showing: i) the clustering pattern (left) among samples from control animals (CTR) and from antibiotic-treated animals following vehicle administration ( $a b x+$ vehicle); ii) the clustering pattern (right) among samples from antibiotic-treated animals subjected to FMT from controls (abx + FMTCTR) or from DNBS-treated animals (abx + FMTDNBS). Samples obtained from control (FMTCTR) or DNBS-treated (FMTDNBS) animals and used for the I and II set of FMT are also shown. The percentage of total variance explained is shown for each component. Taxonomic composition at phylum (B) and family (C) level of samples belonging to the $a b x+$ FMTCTR and to the abx + FMTDNBS group; microbial taxa showing a statistically significant difference (Kruskal-Wallis $\mathrm{P}<0.005)$ in their relative abundance between the two groups are marked with a star $\left(^{*}\right)$ in each legend box. 
Day 18

7 days after the FMT set I

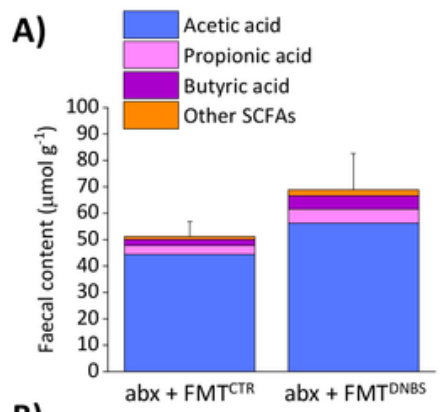

B)

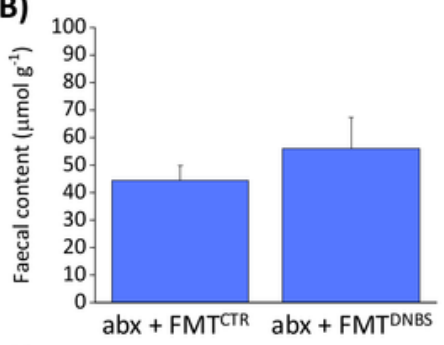

C)
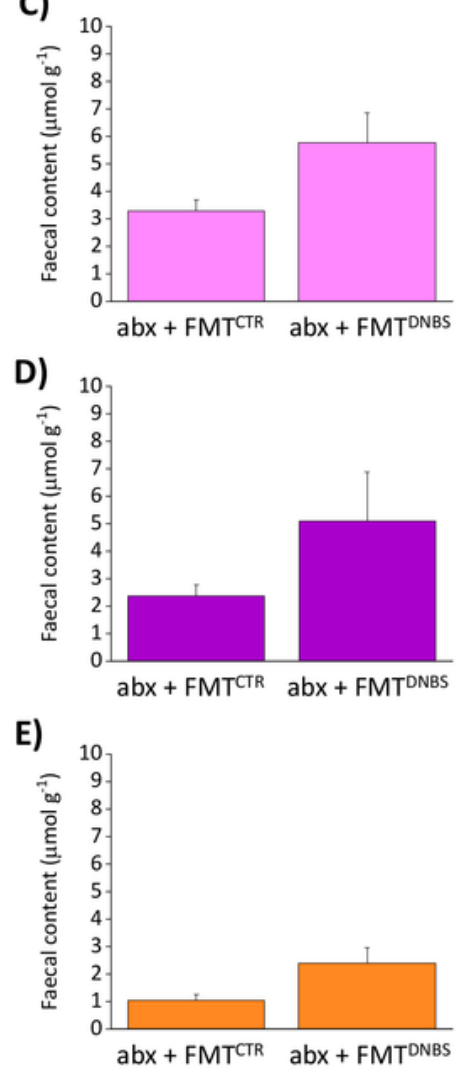

Day 32

7 days after the FMT set II
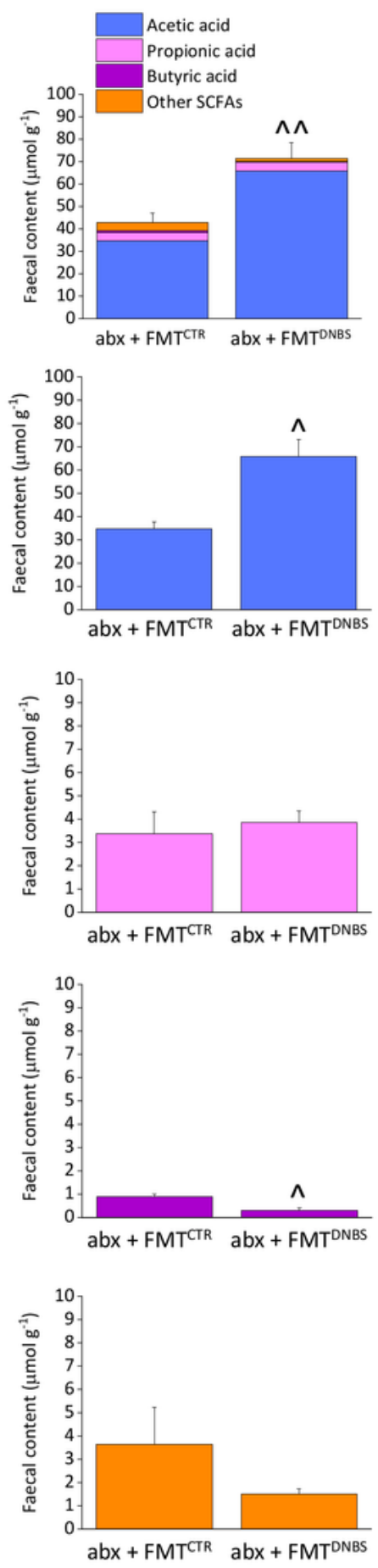

Day 46

21 days after the FMT set II
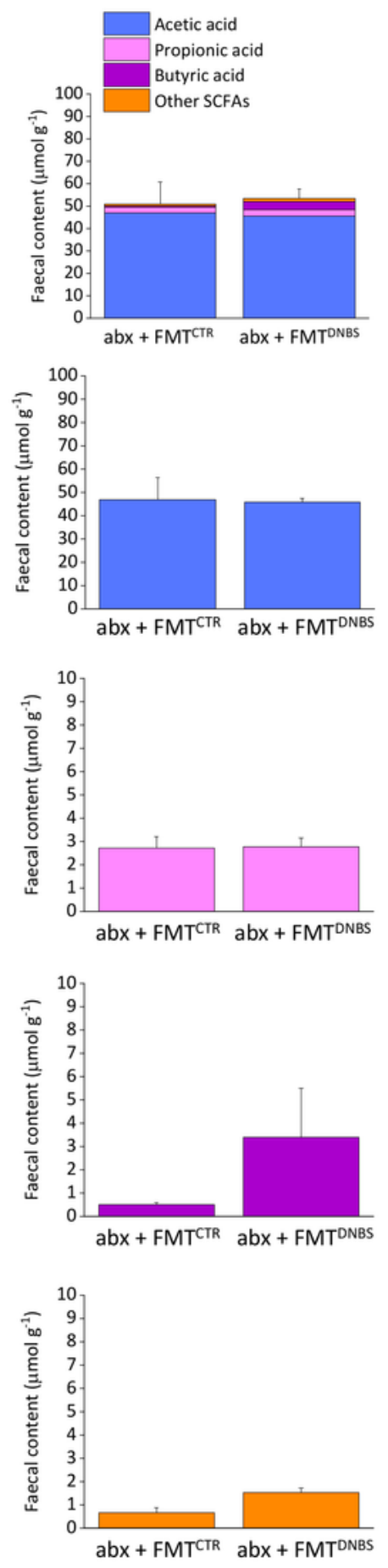

\section{Figure 3}

Effect of FMT on faecal SCFAs concentrations. HPLC analysis of A) total SCFAs, B) acetic acid, C) propionic acid, D) butyric acid and E) other fatty acids concentration in faecal pellets. Each value represents the mean \pm SEM of $5-9$ animals per group. ${ }^{\wedge} \mathrm{P}<0.01$ and ${ }^{\wedge} \mathrm{P}<0.05$ vs abx + FMTCTR treated animals. 
A) Macroscopic Damage Score

Day 7: $24 \mathrm{~h}$ after the end of abx regimen

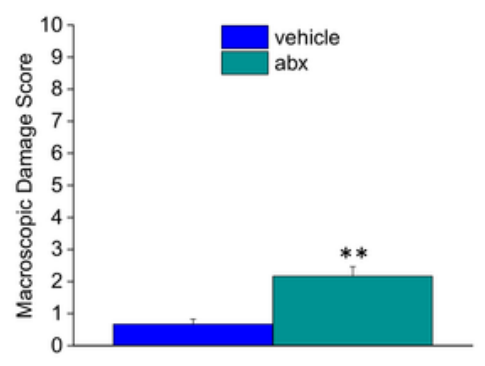

Day 32: 7 days after the FMT set II

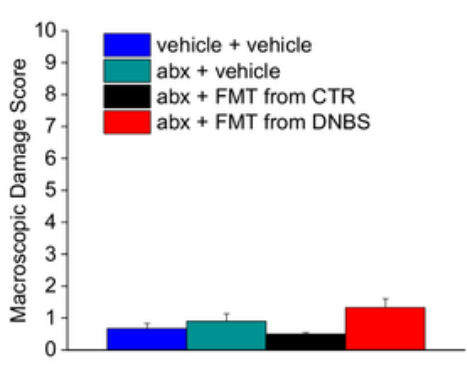

Day 46: 21 days after the FMT set II

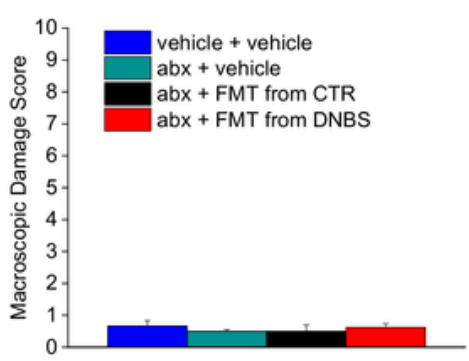

B) Microscopic analysis - H\&E staining
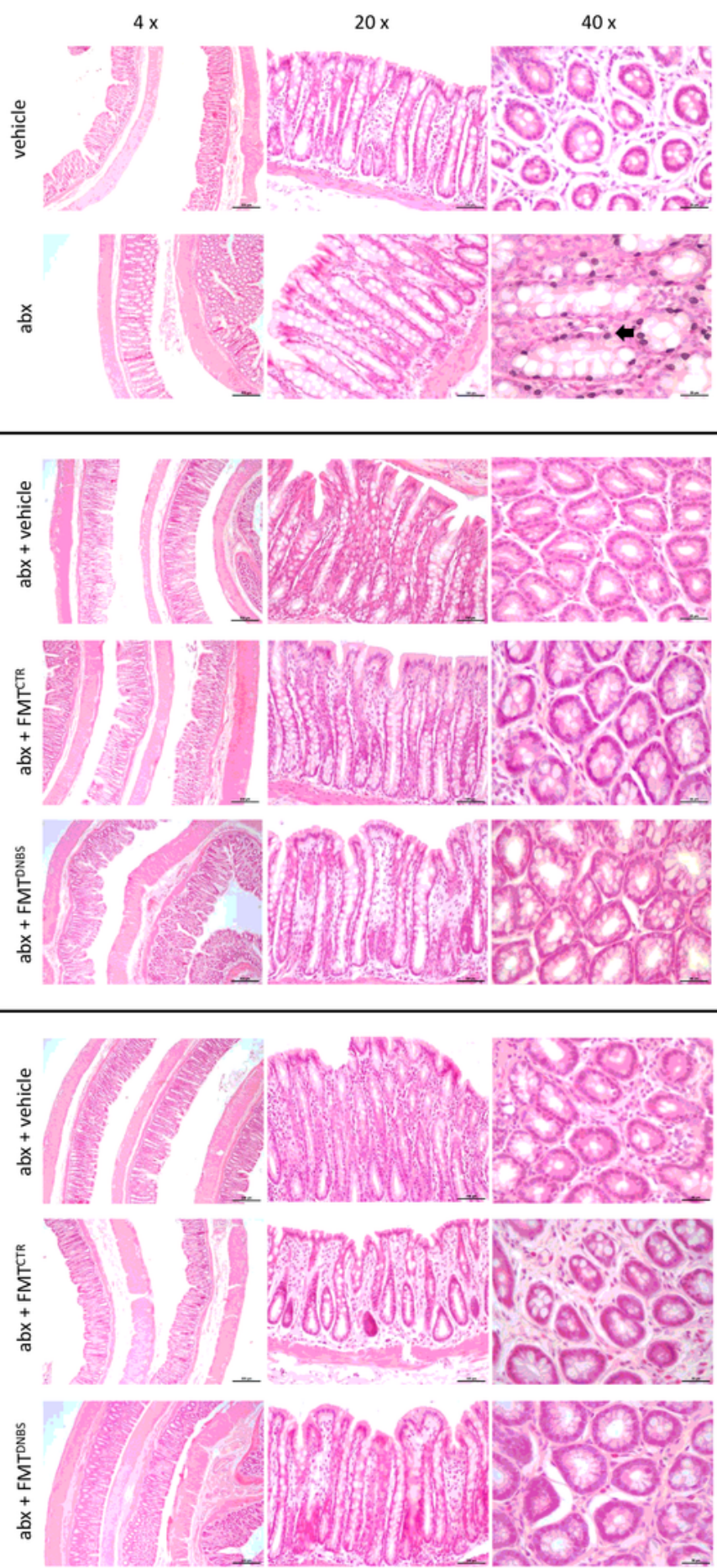

\section{Figure 4}

Histological evaluation of colon damage after the antibiotic regime and the FMT. A) Macroscopic Damage Score; B) Histological analysis on haematoxylin/eosin stained colon slices. Each value represents the mean \pm SEM of $4-6$ animals per group. ${ }^{* *} \mathrm{P}<0.01$ vs vehicle treated animals. 

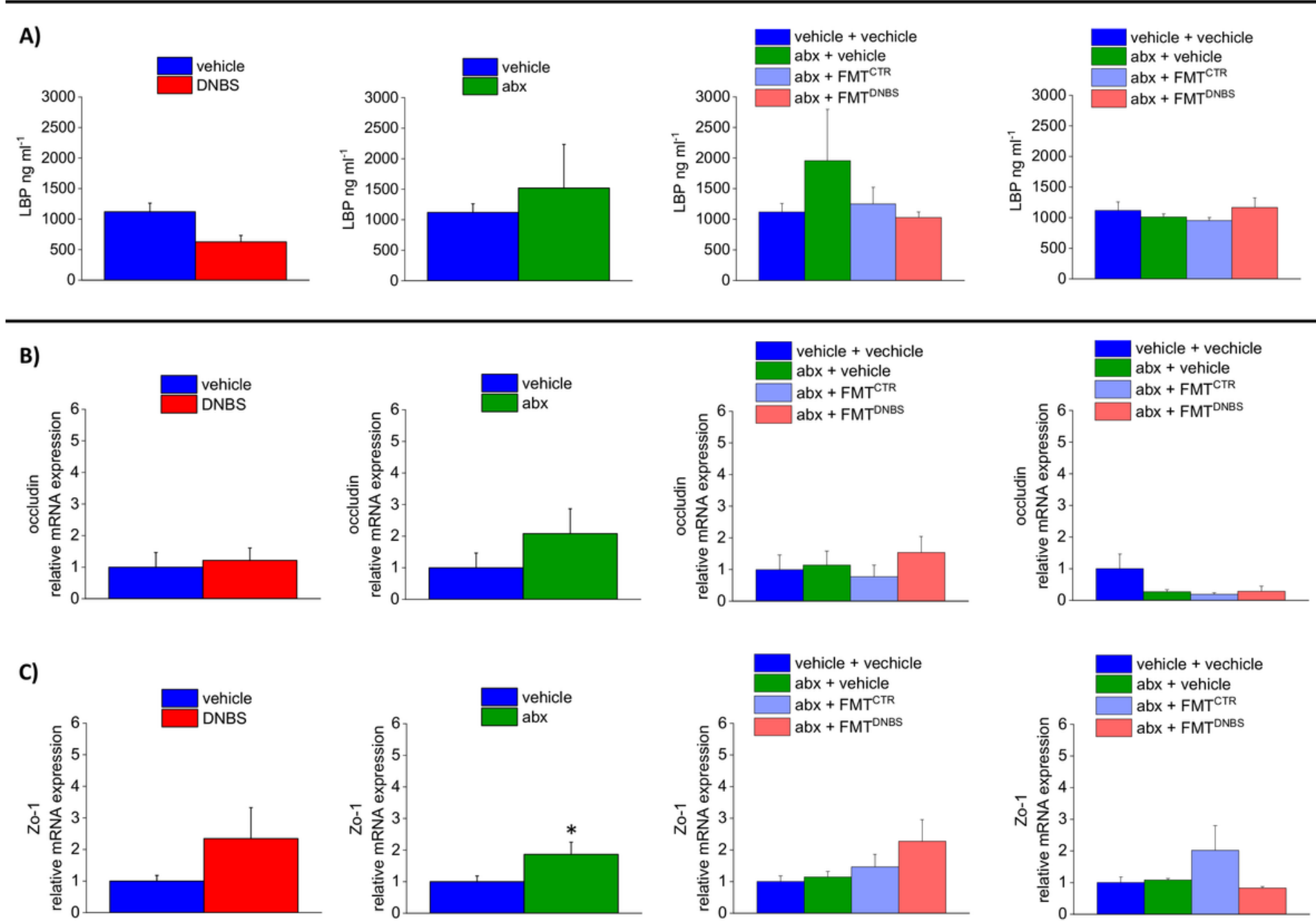

Figure 5

Effect of antibiotic regime and FMT on gut permeability. A) Elisa assay for Lipopolysaccharides Binding Protein (LBP) in plasma samples. Analysis of occludin (B) and Zo-1(C) gene expression on colon samples by RT-qPCR. The mRNA expression was normalized to $\beta$-actin and fold changes were expressed in comparison with control group Each value is the mean \pm S.E.M. of $3-6$ rats per group. ${ }^{*} P<0.05$ and ${ }^{*} \mathrm{P}<0.01$ vs vehicle treated animals. 

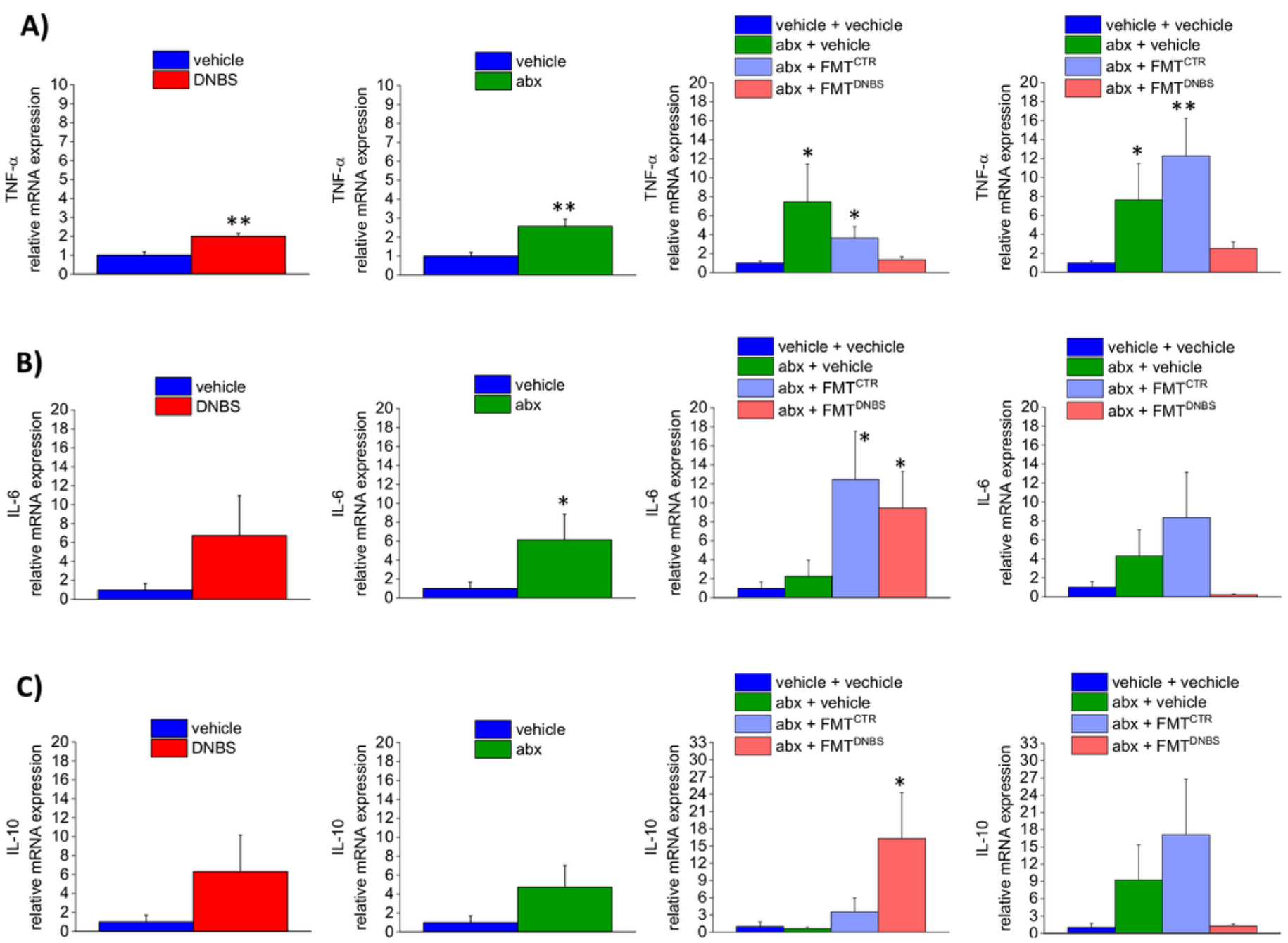

D)
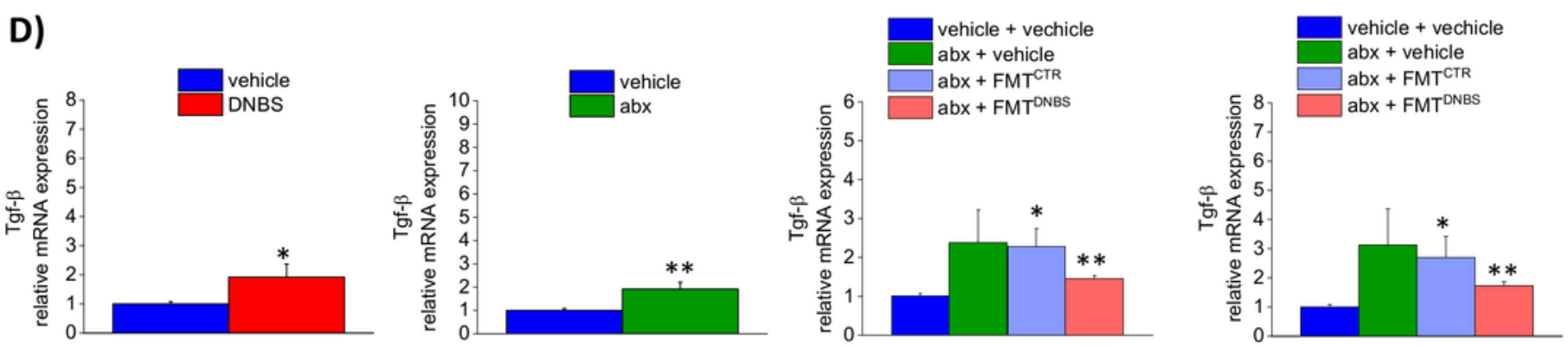

\section{Figure 6}

Effect of antibiotic regime and FMT on gut cytokines profile. Analysis of TNF-a (A), IL-6 (B), IL-10 (C) and TGF- $\beta$ (D) gene expression on colon samples by RT-qPCR. The mRNA expression was normalized to $\beta$ actin and fold changes were expressed in comparison with control group Each value is the mean \pm S.E.M. of 4-6 rats per group. ${ }^{*} \mathrm{P}<0.05$ and ${ }^{*} \mathrm{P}<0.01$ vs vehicle treated animals. 

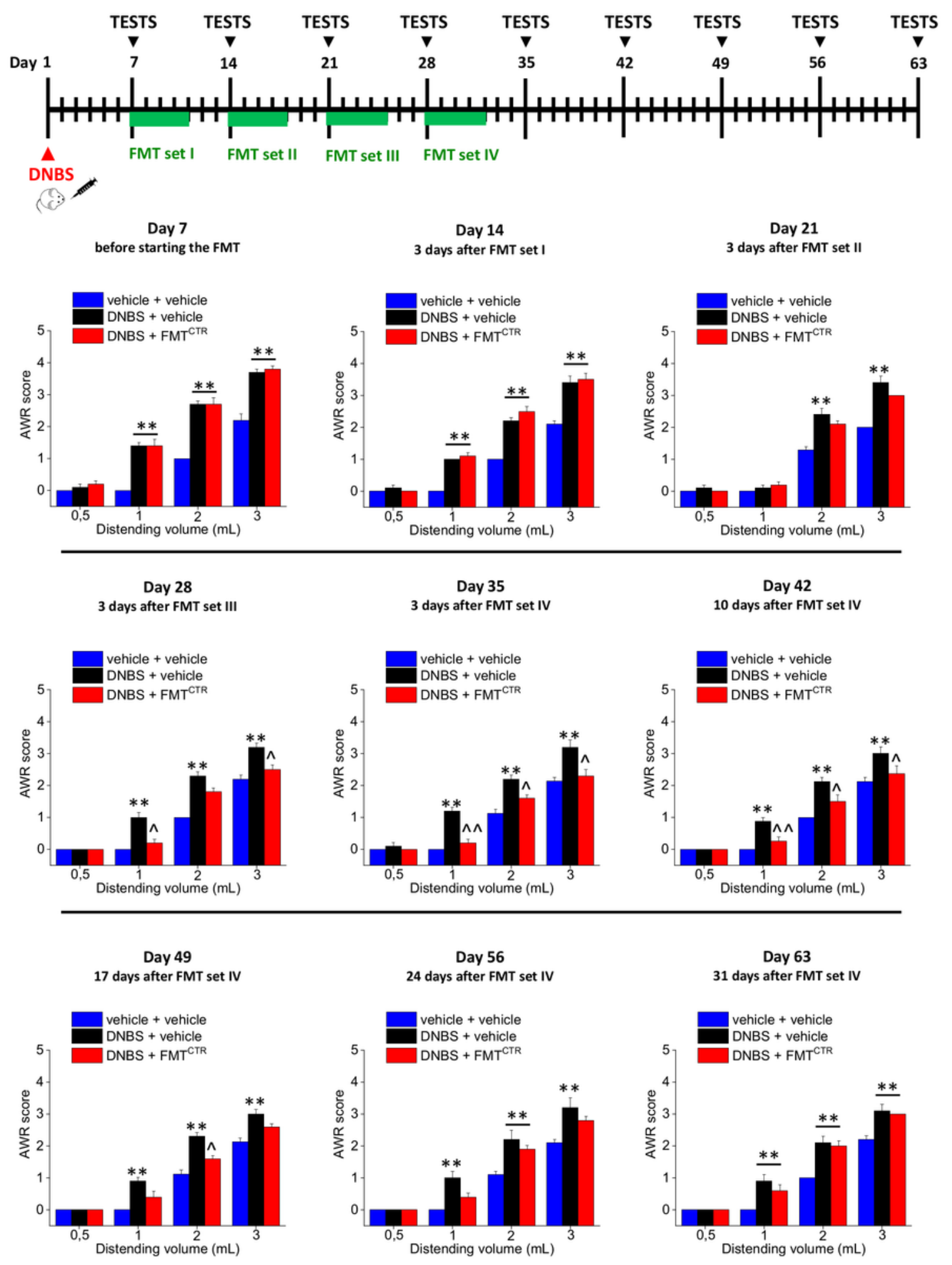

\section{Figure 7}

Therapeutic effect of FMT on DNBS-induced post-inflammatory visceral pain. As shown in the scheme, rats were intra-rectally injected with DNBS (30 mg in $0.25 \mathrm{~mL}$ EtOH $50 \%$ ); on day 7 DNBS-animals were divided into 2 groups respectively administered with the vehicle or the FMT from CTR donors per os for 5 consecutive days. The FMT set was weekly repeated for 4 times. Behavioral tests were performed at the end of the antibiotic treatment, 3 days after each cycle of FMT and once week afterwards. Visceral 
sensitivity was assessed in the animals by measuring the Abdominal Withdrawal Response (AWR) to Colo-Rectal Distension (CRD; $0.5-3 \mathrm{~mL}$ ). Each value is the mean \pm S.E.M. of 5 rats per group. ${ }^{*} P<0.05$ and ${ }^{\star *} \mathrm{P}<0.01$ vs vehicle or vehicle + vehicle treated animals. ${ }^{\wedge} \mathrm{P}<0.05$ and ${ }^{\wedge}{ }^{\wedge} \mathrm{P}<0.01$ vs $\mathrm{DNBS}+$ vehicle treated animals.

\section{Supplementary Files}

This is a list of supplementary files associated with this preprint. Click to download.

- GraphicalAbstract.png

- Supplementarymaterial.docx 